\title{
A NEW TEXTURE SYNTHESIS BASED IMAGE RESTORATION APPROACH WITH IMAGE FILTER
}

\author{
Navdeep Mor ${ }^{1}$, Surinder ${ }^{2}$
}

\begin{abstract}
Inpainting means filling of areas/regions of an image or video in such a way that the modified region(s) is visually agreeable to human eye. Inpainting technique has many applications such as, object removal in digital photos, removal of occlusions (date ,stamps ,logo etc.), such as large unwanted regions, red eye correction, super resolution, restoration of old films and paintings etc. Inpainting can also be use to create special effects by removing unwanted substance from the image. Image inpainting approaches may be roughly divided into two categories: (i) partial differential equation (PDE) based approach for structure propagation and (ii) exemplar-based approach for texture synthesis. In this work we have followed the latter one.

Restoration of a damaged/historical image has always been an important part of image processing and finds use in a surfeit of spheres. This process may be carried out manually or by using some automated techniques. Image inpainting is one such technique which helps us to accomplish these objectives by means of filling up the missing regions in the damaged images. We click many images and always want to keep them preserve for long time. And as the time past, those pictures got damaged (starches, cracks, Scratch, image data loss), the solution is image Inpainting. This technique can restore the lost parts of an image and reconstruct them based on the neighbor's information.
\end{abstract}

Keywords: Inpainting, Filtering, Image Restoration, Biometric, MSE, PSNR.

\section{INTRODUCTION}

In this paper, a novel approach is proposed 'Improved Exemplar Based Image Inpainting Method with Image Filtering Approach'. The proposed methodology aims to use of exemplar based inpainting technique with image filtering process to produce more efficient results and performance.

Inpainting means filling of areas/regions of an image or video in such a way that the modified region(s) is visually agreeable to human eye. Inpainting technique has many applications such as, object removal in digital photos, removal of occlusions (date ,stamps ,logo etc.), such as large unwanted regions, red eye correction, super resolution, restoration of old films and paintings etc. Inpainting can also be use to create special effects by removing unwanted substance from the image. Image inpainting approaches may be roughly divided into two categories: (i) partial differential equation (PDE) based approach for structure propagation and (ii) exemplar-based approach for texture synthesis. In this work we have followed the latter one. This approach may be useful for different biometric system for enhancing the quality of input images [18-24].

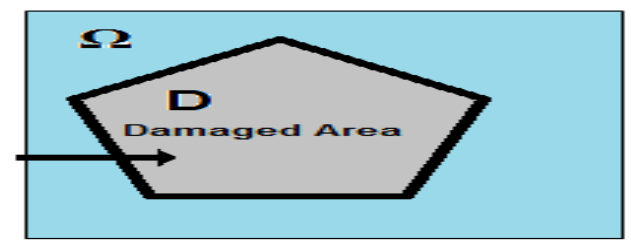

Figure 1: In this picture D is the Damaged region on the basis of known region.

\footnotetext{
${ }^{1}$ Department of Computer Applications Singhania University, Jhunjhunu, Rajasthan, INDIA

${ }^{2}$ Dept. of Computer Science \& Engineering, HCTM Technical Campus, Kaithal (Haryana)
} 
Image inpainting refers to repairing a damaged picture where part of the information has been lost. As shown in Figure 1 , for a corrupted image, where the inpainting domain D is missing and the content outside $\mathrm{D}(\Omega)$ is known, image inpainting is to fill domain D which makes the whole picture "meaningful" and looks like undamaged.

Restoration Process: As we have already discussed using image inpainting we can recover lost or deteriorated portions of pictures by using information from its neighbors. If done manually, than this process can be very dreary and time consuming, for example, the task pertaining to restoring a valuable classic painting, would be assigned to a skilled art conservator. Artists and conservators have been using manual inpainting to revive damaged paintings for ages. But we can improve it, with the help of automation, digital image processing techniques available today, the same task can be carried out more efficiently and effectively in considerably lesser time. As it can be seen in the next image the restoration process [14-17].

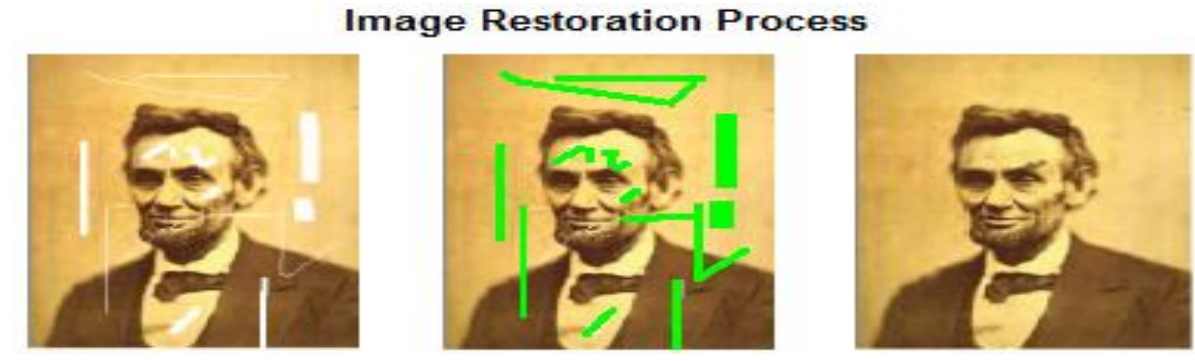

Damaged Image

Masked Image

Inpainted Image

Figure 2: Restoration Basic Technique [2].

We can also use this to obect removal also as it can be seen in the next image where little elephant has been removed from the image.

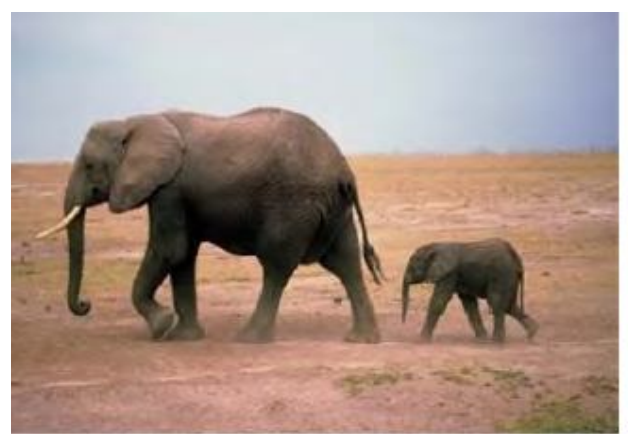

(a)

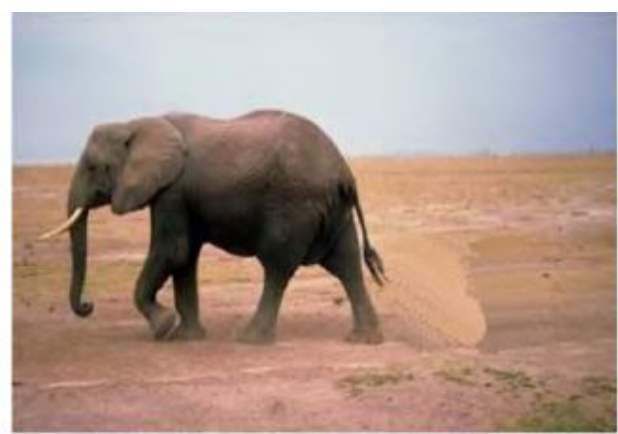

(b)

Figure 3: Object removal Image inpainting (a) Original Image (b) Inpainted Image[3]

\section{REVIEW OF LITERATURE}

Bertalmio et al. (2000) [4] Author proposed a method that attempted to restore the lost regions of damaged images by manually demarcating them by the use of some color.

Shen et al. (2003) [5] aimed at providing an inpainting method that was based upon the Euler Elastica model. They studied the use of the existing models and partial differential equations for curves and their applicability to image inpainting. Euler Lagrange equation for curvature based inpainting was formulated.

Criminisi et al. (2004) [6] provided an algorithm that could remove considerably larger objects from images. The algorithm used exemplar-based texture synthesis which was modulated by a unified approach to decide the sequence of filling target areas but was high on time complexity.

Awate et al. (2006) [7] proposed an unsupervised, information theoretic, adaptive filter (UINTA) that improves predictability of intensities of pixels from their respective neighborhoods by reducing their combined entropy. Thus being nonlinear, nonparametric, adaptive, and unsupervised, it can restore a wide range of images with almost no parameter tuning data.

Ntogas et al. (2008) [8] presented a binarization mechanism for historical manuscripts and images. It divided the process into five distinct steps for the six defined categories of images and applied a refinement procedure on these to 
obtain enhanced results. The improved visual quality and readability of the image texture makes this technique viable and efficient to be used in various applications for preliminary processing of document images.

Zongben Xu et al. (2010) [9] presented exemplar based inpainting by using the natural image patch's sparsity. In contrast to traditional exemplar inpainting this method performs better in terms of distinguishing the structure and the texture and also sharper and consistent inpainted regions are obtained due to sparse representations. The results were demonstrated on both real and synthetic images.

Zhen Xie et al. (2011) [2] compared and analyzed four existing inpainting algorithms and provided a novel inpainting algorithm that divided the damaged image into blocks with specific traits. The adaptive matching algorithm proposed could automatically choose suitable methods to inpaint every block to generate optimal results. They experimentally proved their algorithm could inpaint more effectively and efficiently.

Sandhya N. et al. (2012) [10] analyzed the various kinds of noise that are present in historical documents that are based on Kannada, the predominant language in the state of Karnataka, India. Each noise is categorized by its source, obstacles posed to character recognition and the effects they have. The major types of noise identified in in historical documents are: a) border, b) skew, c) noisy background, d) touching characters and e) degraded characters.

Li et al. (2014) [1], [11] proposed an algorithm that uses compressed sensing (CS) in the frequency domain in order to recover damaged images. The image is disintegrated into two functions - structure and textual parts. The method ensured a decent restoration of the structure and quality of the image. The PSNR is generally high and the method has an edge in terms of time complexity.

Kawade M.D et al. (2014) [3] proposed a technique to remove multiple object removal using adaptive patch selection. The method first divides the image into target region and the source region and then locates the best matching source patch based on the target region's surrounding pixels. It provides a better result in terms of its execution time and PSNR.

\section{OBJECTIVES OF THE STUDY}

Image inpainting is the procedure of restoring/reconstruction and regenerating unknown regions in the image from the known regions by using their available information. There are many inpainting algorithms however each method has its own set of advantages and shortcomings. Majority of the techniques are working well but my goal is to address the issue of an image by laying emphasis on defects and degradations that are peculiar to such images. The images may contain damages such as: Scratches, Holes, Cracks, Water effects and unwanted marks on the image. The objectives of our work will be developing an improved algorithm having a:

- Remove of all types of damages (Scratches/holes/cracks) and reconstruct it.

- Better performance in terms of time, MSE, PSNR values compare to previous exampler based method

- Good visual quality.

\section{PROPOSED WORK}

We know that to remove any kind of noise filter may be used. S o here to improve the results of previous algorithms I will try some filters. After apply the filters the images will be given to the inpainting method with the damaged image and the results will be better in terms of time, MSE and PSNR values as describe via the next proposed flowchart. Let us see the designed algorithm to achieve these objectives.

\section{Algorithm:}

Step: 1 Load Images

Load Reference Image

Load Corrupted Image

Load Mask Image

Step: 2 Apply filter to Original image

Use filter (wiener2) to the original image to improve the visual clarity

Step: 3 Call Restoration Method

Inpainting(Dimg,Mimg)

Dimg= Damaged Image, Mimg= Masked Image

Step: 4 Show the Output

Confidence Term, Data Term and, Reconstructed Image

Step: 5 Compare and evaluate the Results

Comparative results in respect to MSE and PSNR

Graphically Presentation of Results 


\section{RESEARCH METHODOLOGY}

- Filling-in the spoiled region is done according to the global features which helps preserving the texture

- Continuing structural local properties from the source area into the damaged area which allows achieving better results in terms of the restored image perceived visual quality with the enhanced edges.

- For pursuing this hybrid approach classifier SVM is proposed in order to avoid consideration of non-useful parts of the image for inpainting, thereby reducing the processing time.

\section{EXAMPLER BASED TEXTURE SYNTHESIS APPROACH}

This algorithm is based on two fundamental principles. First, exemplar-based texture synthesis is sufficient to propagate extended linear structures and an additional synthesis mechanism is not required for handling isophotes (line of equal gray values). Second, the order of filling patches is of fundamental importance. [12,13].

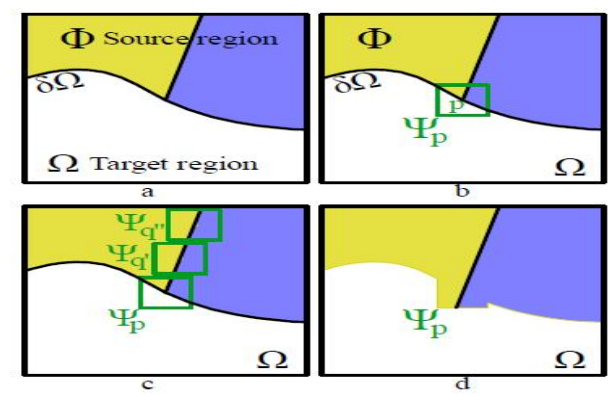

Figure 8: (a) Original image and target region $\Omega$, source region $\Phi$, (b) The patch $\Psi$ p inpainted area (c) Similar candidate patches for the source region, (d) The best matched patch has been copied into $\Psi \mathrm{p}$ [12]

\section{a) Noise Removal Filter}

wiener2 filter which is a 2-D adaptive noise-removal filtering. wiener2 lowpass-filters a grayscale image that has been degraded by constant power additive noise. wiener2 uses a pixel wise adaptive Wiener method based on statistics estimated from a local neighborhood of each pixel.

\section{Syntax}

$J=$ wiener $2(I,[m n]$, noise $)$

$[J$, noise $]=$ wiener $2(I,[m n])$

The input image I is a two-dimensional image of class uint8, uint16, int16, single, or double. The output image $\mathbf{J}$ is of the same size and class as $\mathbf{I}$.

\section{b) SDK}

A software development kit (SDK or devkit) is typically a set of software development tools that allows the creation of applications for a certain software package, software framework, hardware platform, computer system, video game console, operating system, or similar development platform.

\section{CONCLUSIONS}

Restoration of a damaged/historical image has always been an important part of image processing and finds use in a surfeit of spheres. This process may be carried out manually or by using some automated techniques. Image inpainting is one such technique which helps us to accomplish these objectives by means of filling up the missing regions in the damaged images. We click many images and always want to keep them preserve for long time. And as the time past, those pictures got damaged (starches, cracks, Scratch, image data loss), the solution is image Inpainting. This technique can restore the lost parts of an image and reconstruct them based on the neighbor's information.

We can say that, in the last means after implementation of our proposed work, the results will definitely be better than previous exampler method. To observe difference and to compare the performances of both methods a comparatively list will be made and the graphs will be generated. The comparison criteria will be MSE, PSNR and the quality of resulted image.

\section{REFERENCES}

[1] Chuang Zhu, Huizhu Jia, Meng Li "Highly Efficient Local Non-Texture Image Inpainting Based on Partial Differential Equation”, 2014 IEEE 17th International Conference on Computational Science and Engineering. 
[2] Zhen Xie; Fan Zhang; Conggui Zhang, "An adaptive matching algorithm for image inpainting,", International Conference on Electronics, Communications and Control (ICECC), vol., no., pp.1293,1296, 9-11 Sept. 2011.

[3] Prof. Kawade M.D., Prof. Ufade A.S., 'Multiple Object Removal Using Exemplar based Image Inpainting'. International Journal of Computer Science \& Engineering Technology, Volume 5, Issue 5, pp. 488-493, May 2014.

[4] M. Bertalmio, J. Sapiro, V. Caselles, et al. "Image Inpainting". In: Proceedings of SIGGRAPH[C], New Orleans, USA, 2000: $417 \sim 424$.

[5] Shen, Jianhong, Sung Ha Kang, and Tony F. Chan. 'Euler's Elastica And Curvature-Based Inpainting'. SIAM J. Appl. Math. 63.2 (2003): 564-592.

[6] Criminisi, A., P. Perez, and K. Toyama. 'Region Filling And Object Removal By Exemplar-Based Image Inpainting'. IEEE Trans. on Image Process. 13.9 (2004): 1200-1212.

[7] Awate, S.P.; Whitaker, R.T., "Unsupervised, information-theoretic, adaptive image filtering for image restoration," Pattern Analysis and Machine Intelligence, IEEE Transactions on Pattern Analysis and Machine Intelligence (TPAMI), vol.28, no.3, pp.364,376, March 2006.

[8] Ntogas, Nikolaos and Ventzas, Dimitrios, "A Binarization Algorithm for Historical Manuscripts", International Conference on Communications, pp. 41-51, 2008.

[9] Zongben Xu, and Jian Sun. 'Image Inpainting By Patch Propagation Using Patch Sparsity'. IEEE Tranactions on Image Processing. 19.5 (2010): 1153-1165.

[10] Sandhya N, R. Krishnan and D. R. Ramesh Babu, "A language independent Characterization of Document Image Noise in Historical Scripts", International Journal of Computer Applications, Vol. 50, No. 9, pp. 11-18, 2012.

[11] Li, Q., Han, Y., \& Dang, J. "Image decomposing for inpainting using compressed sensing in DCT domain", 2014 Frontiers of Computer Science, Volume 8, Issue 6, pp 905-915 , 905-915.

[12] A. Criminisi, P. Pérez, and K. Toyama, "Region filling and object removal by exemplar-based image inpainting," IEEE Trans. Image Process., vol. 13, pp. 1200-1212, 2004.

[13] A. A. Efros and T. K. Leung, "Texture synthesis by non-parametric sampling," in IEEE Conf. Computer Vision, 1999, pp. 1033-1038.]

[14] Neha Khanna, Dr. Pankaj, “A New Texture Synthesis Based Image Restoration Approach With Image Filter” ISSN : 23492819, Volume 3, Issue 2, Feb-2016.

[15] S. Jangra, S. Goel and A. Selwal, "Hyper Spectral Image Restoration Approach using LRMR and LDA", IEEE Xplorer, Pg. 415-418, 2015, e-ISBN: 978-1-5090-0148-4.

[16] T. Kumar, S. Jangra and S. Bhushan, "Face Recognition with Decision Tree using SVM and SURF", Published in, "Int'1 J. of Control Theory and Applications", Vol. 10 (2017), Issue No. 15, Pg. 173-180, ISSN: 0974-5572.

[17] Y. S. Sangwan, S. Jangra and K. Kuhar, "Facial Skin Segmentation using BFO and PSO”, Int'1 J. of Control Theory and Applications, Vol. 10 (2017), Issue No. 18, Pg. 31-39, ISSN: 0974-5572.

[18] A. Selwal, S.K Gupta and S. Jangra, “A Hybrid Template Security Scheme for Multimodal Biometric System based on Fingerprint and Hand Geometry", Int'1 J. of Control Theory and Applications, Vol. 10 (2017), Issue No. 15, Pg. 143-152, ISSN: 0974-5572.

[19] Shant Kaushik and Surender Jangra, "Finger Vein Biometric Authentication Scheme using Repeated Line Tracking and DWPT based Scheme", Int'1 J. of Control Theory and Applications, Vol. 10 (2017), Issue No. 18, Pg. 289-294, ISSN: 0974-5572.

[20] A. Selwal, S.K Gupta and Surender, "Low Overhead Octet Indexed Template Security Scheme for Multimodal Biometrics System”, Journal of Intelligent \& Fuzzy System 32 (2017), pp. 3325-3337, DOI:10.3233/JIFS169274, IOS Press.

[21] A. Selwal, S.K. Gupta and S. Kumar, "A Scheme for Template Security at Feature Fusion Level in Multimodal Biometric System”, Advances in Science and Technology Research Journal, Vol. 10, No. 31, Sept. 2016 , Pg. 23-30.

[22] A. Selwal, S.K. Gupta and Surender, "Fuzzy Analytic Hierarchy Process based Template Data Analysis of Multimodal Biometric Conceptual”, Procedia Computer Science (Elsevier), 85 (2016), Pg. 899-905.

[23] A. Selwal, S.K. Gupta and Surender, "Template Security Analysis of Multimodal Biometric Frameworks based on Fingerprint and Hand Geometry", Perspectives in Science (Elsevier), (2016) 8, Pg.705-708, ISSN: 2212-0209.

[24] A. Selwal, S. K. Gupta, Surender and Anubhuti, "Performance analysis of template data security and protection in Biometric Systems, IEEE Xplore, 2015, pp. 1-6. e-ISBN : 978-1-4673-8253-3. 